\title{
Differential Responses in Human Striatum and Prefrontal Cortex to Changes in Object and Rule Relevance
}

\author{
Roshan Cools, Luke Clark, and Trevor W. Robbins \\ Department of Experimental Psychology, University of Cambridge, Cambridge, CB2 3EB, United Kingdom
}

\begin{abstract}
Event-related functional magnetic resonance imaging was used to measure blood oxygenation level-dependent responses in 16 young healthy human volunteers during performance of an attentional switching task. The task allowed the separate investigation of lowerorder switching between concrete objects and higher-order switching between abstract task rules. Significant signal change in the ventral striatum was demonstrated on trials when subjects switched between objects but not when subjects switched between abstract task rules. In contrast, signal change in the lateral prefrontal cortex $(\mathrm{PFC})$ was observed during all switch trials. The switch-related responses were not contaminated by task difficulty, because the greatest signal change was observed during the relatively easy switch trials, which required both lower-order and higher-order switching at the same time. The present data suggest that mechanisms of inhibitory response control in frontostriatal systems are organized according to distinct levels of abstraction. Specifically, the response selection computation carried by the ventral striatum, which projects to the orbitofrontal cortex and the medial PFC, is restricted to the transformation of concrete stimulus exemplar information into motor responses, whereas the adaptive function of the lateral PFC extends to the transformation of abstract task-rule representations into action.
\end{abstract}

Key words: object alternation; inhibitory control; task switching; PFC; striatum; adaptation; attention; basal ganglia; cognitive; frontal; imaging

\section{Introduction}

Inhibitory control functions have been most commonly associated with the prefrontal cortex (PFC). In particular, lesions of the orbitofrontal cortex (OFC) and the ventrolateral PFC (VLPFC) in animals disrupt performance on tasks including reversal learning and object alternation (Mishkin et al., 1969; Iversen and Mishkin, 1970; Jones and Mishkin, 1972; Chudasama and Robbins, 2003), leading to the hypothesis that the ventral PFC is critical for inhibitory control (Fuster, 1989).

However, recent evidence suggests that inhibitory control mechanisms are not specific to the ventral PFC but are present throughout the PFC and may be organized according to distinct levels of abstraction (Milner, 1964; Dias et al., 1996; Brown and Bowman, 2002). One study revealed a double dissociation in which lateral PFC lesions disrupted higher-order attentional shifting between abstract stimulus dimensions, whereas OFC lesions disrupted lower-order reversal learning between specific stimulus exemplars (Dias et al., 1996). Consequently, Roberts and Wallis (2000) proposed that the lateral PFC is involved in inhibitory control on the basis of higher-order rules (e.g.,

Received Sept. 22, 2003; revised Dec. 1, 2003; accepted Dec. 1, 2003.

This work was supported by a Wellcome Trust programme grant (T.W.R., L.C.) and completed within the Medical Research Council Centre for Behavioral and Clinical Neuroscience. R.C. is a Royal Society Dorothy Hodgkin fellow and a junior research fellow at St. John's College (Cambridge, UK). We thank Gregory Digirolamo and Matthew Brett for helpful discussion and advice and Ruth Bisbrown-Chippendale and Claire Sleator from the Wolfson Brain Imaging Centre (Cambridge, UK) for radiographic assistance with this study.

Correspondence should be addressed to Roshan Cools, University of California, Department of Psychology, 3210 Tolman Hall, Berkeley, CA 4720-1650. E-mail: roshanco@uclink.berkeley.edu.

DOI:10.1523/JNEUROSCI.4312-03.2004

Copyright $\odot 2004$ Society for Neuroscience $\quad$ 0270-6474/04/241129-07\$15.00/0 stimulus-dimension rules), whereas the OFC is involved in lower-order rule learning. On the basis of the same data, O'Reilly et al. (2002) hypothesized that abstract dimensional information is encoded in the dorsolateral PFC (DLPFC), whereas specific featural information is encoded in the OFC.

Although the role of the striatum in inhibitory control has not generally been emphasized, much evidence suggests that impairments after damage to specific areas within the PFC may overlap with those seen after damage to connected parts of the striatum (Rosvold, 1972; Alexander et al., 1986). For example, reversal learning is disrupted not only by damage to the OFC but also by damage to the ventral striatum (VS) (Divac et al., 1967; Stern and Passingham, 1995), which receives its frontal input primarily from the OFC and the medial PFC (Haber et al., 2000).

Two recent functional imaging studies compared lower-order reversal shifts with higher-order extradimensional shifts (Rogers et al., 2000; Nagahama et al., 2001). However, neither study revealed significant effects in the PFC during lower-order shifts when compared directly with extradimensional shifts. The positron emission tomography (PET) technique, used by Rogers et al. (2000), may have obscured activation because of averaging over an extended period of scanning, and Nagahama et al. (2001) did not acquire data from the ventral brain below the anterior commissure-posterior commissure axis $(z=0)$. Moreover, both studies used higher-order extradimensional shift tasks, which required not only the selection of newly relevant abstract stimulus dimensions among competing alternatives but also the selection of newly relevant specific stimulus exemplars.

Therefore, the hypothesis that inhibitory control within human frontostriatal systems is organized according to distinct lev- 


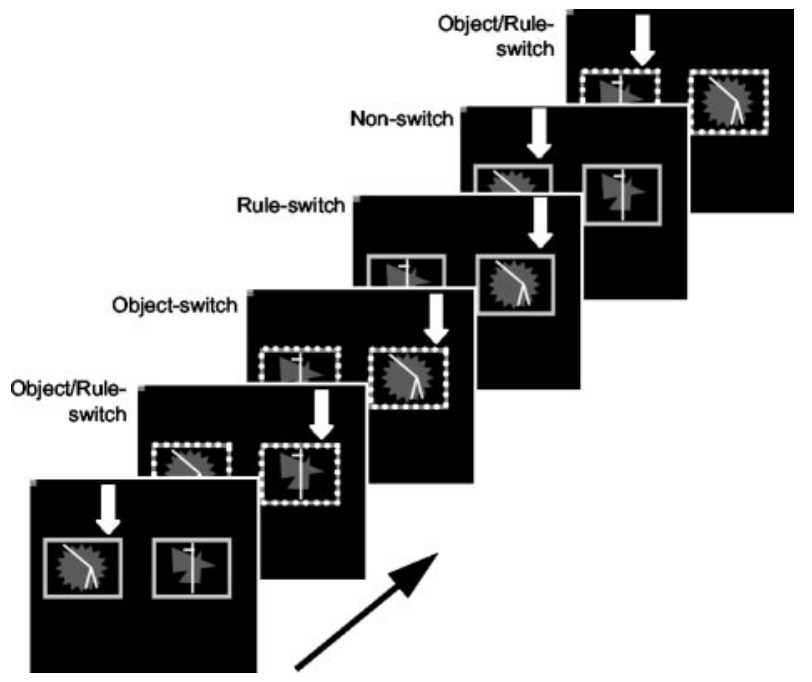

Figure 1. Task design. An example sequence of trials is displayed, with the arrow indicating the correct response. The yellow (here, light gray) stimulus windows cued the subject to choose the same object as on the previous trial. The blue (here, dotted dark gray) stimulus windows cued the subject to switch to the other object. Examples of all four trial types are shown.

els of abstraction has not been tested directly using event-related functional magnetic resonance imaging (fMRI). The current study was designed to test the hypothesis that dorsolateral frontostriatal circuitry is involved in the inhibition-selection of abstract task-rule information, whereas ventral frontostriatal circuitry (including the ventrolateral and orbital PFC) is involved in the inhibition-selection of specific (concrete) stimulus information (i.e., objects). The use of a task in which object-switch trials did not involve any form of rule switching and vice versa enabled us to examine the unique contributions of frontostriatal regions to object switching and rule switching.

\section{Materials and Methods}

Subjects. Sixteen right-handed, young healthy volunteers (eight males and eight females; mean age, 25.4; SD, 7; age range, 18-45) with no history of psychiatric or neurological disorders participated in this study. All subjects gave written informed consent, and the study was approved by the local research ethics committee.

Experimental design. On each trial, the same two abstract colored patterns were presented simultaneously in the left and right visual fields (location randomized) (Fig. 1), and subjects were required to choose one of the two patterns on each trial. Responses were made according to one of two response rules using the left or right button on a button box positioned on the stomach of the subject. The patterns were presented within and at the same time as either blue or yellow stimulus windows. If the windows were blue, subjects were required to choose the pattern that they did not choose on the previous trial (i.e., they switched responding from target stimulus A to target stimulus B). If the windows were yellow, subjects were required to choose the same stimulus as on the previous trial (i.e., the target stimulus remained the same). These rules also applied to trials after an incorrect response, because pilot testing revealed that this procedure was least confusing for subjects. The design allowed us to separate four trial types: (1) trials on which both the task rule and the target object were the same as on the previous trial [i.e., yellow trials after yellow trials (nonswitch trials)], (2) trials on which the task rule but not the target object remained the same [i.e., blue trials after blue trials (object-switch trials)], (3) trials on which the task rule was different from the previous trial but the target object remained the same [i.e., yellow trials after blue trials (rule-switch trials) ], and (4) trials on which both the task rule and the target object were different from the previous trial [i.e., blue trials after yellow trials (object-rule-switch trials)] (Fig. 1).

Each subject performed four blocks of 114 trials (6.3 min), and stimuli were presented in a pseudorandom fixed order so that (1) rule-switching was unpredictable (the probability of a rule-switch was 0.5 on each trial), (2) the number of object-repetition and object-switching trials was matched within each block, and (3) response (location) repetition was approximately matched between the four trial types. Stimuli and cue windows were presented for $2000 \mathrm{msec}$ or until a response was made. If a response was not made within $2000 \mathrm{msec}$, a "too late" message was presented. Feedback, consisting of a green smiley face for correct responses or a red sad face for incorrect responses, was presented immediately after the response. The feedback faces were presented centrally between the two stimuli for $500 \mathrm{msec}$ during which the stimuli also remained on the screen. After feedback, the stimuli were removed, and the face was replaced by a fixation cross for a variable interval so that the overall interstimulus interval was $3.32 \mathrm{msec}$, enabling precise desynchronization from the repetition time (TR) (of $1600 \mathrm{msec}$ ) and sufficient sampling across the hemodynamic response function. Before entering the scanner, subjects performed two practice blocks on average, and these blocks were identical to the first two blocks of trials in the scanner.

The task was programmed in Microsoft (Seattle, WA) Visual Basic 6.0, and stimuli were presented on a computer display projected onto a mirror in the MRI scanner.

Imaging acquisition. Imaging data were collected using a Bruker Medspec scanner (S300; Bruker, Ettlingen, Germany) operating at 3 tesla. In each session, $250 \mathrm{~T} 2{ }^{\star}$-weighted echoplanar images depicting blood oxygenation level-dependent (BOLD) contrast were acquired [TR, $1.6 \mathrm{sec}$; echo time, $27.5 \mathrm{msec}$ ]. Twenty-one slices (each $4 \mathrm{~mm}$ thick; interslice gap, $5 \mathrm{~mm}$; matrix size, $90 \times 90$; bandwidth, $143 \mathrm{kHz}$; acquisition orientation, axial oblique) per image were acquired. The first 12 echoplanar images in each session were discarded to avoid T1 equilibrium effects. Susceptibility artifacts in nasal sinuses led to some signal dropout from the ventromedial PFC and temporal poles.

Imaging analysis. Data analysis was performed using SPM99 and SPM2 (Wellcome Department of Cognitive Neurology, London, UK). Preprocessing procedures included (linear) slice acquisition time correction, within-subject realignment (SPM2), geometric undistortion using fieldmaps (Cusack et al., 2001), spatial normalization (see below), and spatial smoothing using a Gaussian kernel $(10 \mathrm{~mm}$ full width at halfmaximum). Spatial normalization was performed as follows. The mean undistorted functional image was coregistered to the individual subject's spoiled gradient-recalled acquisition in a steady state (the skull was stripped using the brain extraction tool) (Smith, 2002), which was subsequently normalized using SPM2 to the Montreal Neurological Institute (MNI) skull-stripped structural template. The obtained normalization parameter set was then written to the functional images. Time series were high-pass filtered (120).

A canonical hemodynamic response function was used as a covariate in a general linear model, and a parameter estimate was generated for each voxel for each event type (SPM99). The parameter estimate, derived from the mean least-squares fit of the model to the data, reflects the strength of covariance between the data and the canonical response function for a given condition. Individuals' contrast images, derived from pair-wise contrasts between parameter estimates for different events, were taken to a second-level group analysis in which $t$ values were calculated for each voxel, treating intersubject variability as a random effect. The $t$ values were transformed to unit normal $\mathrm{Z}$ distribution to create a statistical parametric map for each of the planned contrasts (described below).

The hemodynamic response function was modeled to the presentation of the stimulus, which co-occurred with the presentation of the cue. The following events were modeled (Fig. 1): (1) nonswitch trials, (2) objectswitch trials, (3) rule-switch trials, and (4) combined object-rule-switch trials. The object-switch trials and rule-switch trials were chosen as critical events of interest, and the two contrasts of interest were object switch minus rule switch and vice versa. The first trial in each block, error trials (including omissions and premature responses), and trials after such error trials were not included in the model.

We predicted significant signal change in the VS and ventrolateral and orbital PFC during object-switch trials relative to rule-switch trials. Conversely, we predicted significant signal change in the dorsal striatum and 


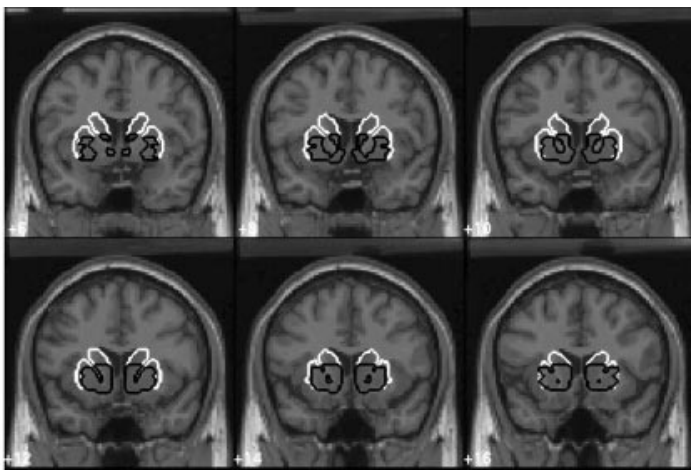

Figure 2. Regions of interest. The ventral striatum is shown in black, and the dorsal striatum is shown in white. [This figure is based on work by Tzourio-Mazoyer et al. (2002)].

dorsal PFC during rule-switch trials relative to object-switch trials. These a priori predictions justified application of region of interest (ROI) analyses. The ventral and dorsal striatal ROIs were drawn directly on top of the "AAL" ROIs published by Tzourio-Mazoyer et al. (2002) (Fig. 2). These ROIs were based on work by Haber et al. (2000) and Groenewegen et al. (1997). Although there is considerable agreement about the anatomical boundaries of and within the striatum, a clear definition of both anatomical and functional subregions within the PFC is lacking. Therefore, prefrontal ROIs were derived from the data themselves. To obtain prefrontal activation clusters that were orthogonal to our comparisons of interest between object-switch and rule-switch trials, a third contrast was calculated in which the combined object-rule-switch event was contrasted with the nonswitch event. This contrast revealed significant peaks in the VLPFC [Talairach coordinates (Talairach and Tournoux, 1988) $(x$, $y, z=-30,27,6$ [Brodmann's area (BA) 11 or 47]; $t=9.59 ; p=0.002$; volume, $\left.108 \mathrm{~mm}^{3}\right)$ and $(x, y, z=-42,15,6[\mathrm{BA} 45] ; t=8.14 ; p=0.015$; volume, $\left.\left.189 \mathrm{~mm}^{3}\right)\right]$, the posteroventral PFC $[x, y, z=-42,9,21$ (BA 44); $t=8.57 ; p=0.008$; volume, $\left.432 \mathrm{~mm}^{3}\right]$, the DLPFC $[x, y, z=-48,24,21$ (BA 9 or 45$) ; t=7.51 ; p=0.037$; volume, $\left.27 \mathrm{~mm}^{3}\right]$, and the anterior cingulate cortex $[x, y, z=9,9,48$ (BA 32); $t=9.77 ; p=0.001$; volume, $1026 \mathrm{~mm}^{3}$ ]. No other peaks were observed within the PFC. Peaks outside of the PFC were observed in the superior parietal cortex $[x, y, z=-27$, $-51,48$ (BA 7)], the ventromedial striatum $(x, y, z=9,-3,-3)$, the premotor cortex $[x, y, z=-45,0,33$ (BA 6) and $x, y, z=24,0,54$ (BA 6)], and the occipital cortex $[x, y, z=-33,-87,0$ (BA 18) and $x, y, z=15$, $-66,12$ (BA 17)]. The frontal activation clusters were thresholded at $p<$ 0.05 (corrected for multiple comparisons at the whole-brain level) and selected directly for subsequent ROI analysis. The total number of ROIs came to nine; therefore, the significance threshold for ROI analyses was set at $p=0.05 / 9=0.006$.

The statistical model described above was then reapplied to the average signal within the ROIs using the MarsBar tool (Brett et al., 2002).

Behavioral analysis. The first trial in each block, incorrect trials, trials on which subjects did not respond within the maximum of $2000 \mathrm{msec}$ (omissions), premature responses $(<300 \mathrm{msec})$, and trials after errors and omissions were all excluded from reaction time (RT) analyses. All 16 subjects performed well on the task, and individual percentages of errors and omissions did not exceed 9 and 1.5\%, respectively. Mean proportions of errors and omissions were $\arcsin$-transformed [2arcsin $\sqrt{ } x$ (Howell, 1997)], whereas mean RTs were log10-transformed. Data were analyzed using repeated-measures ANOVAs (version 11; SPSS, Chicago, IL) with one within-subject factor (trial; four levels) and a priori defined repeated within-subjects contrasts (nonswitch versus object switch, object switch versus rule switch, and object switch versus object-rule switch).

\section{Results}

\section{Imaging data}

Whole-brain contrast images with continuous activation values are shown in Figure 3, $a$ and $b$, effects are plotted in Figure 4, and

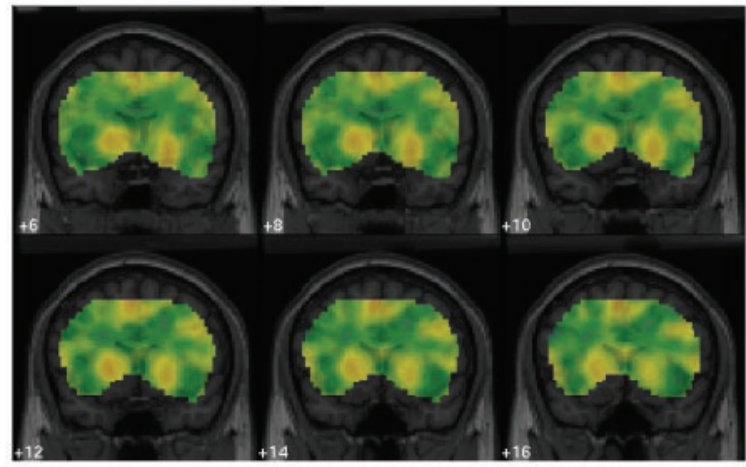

B

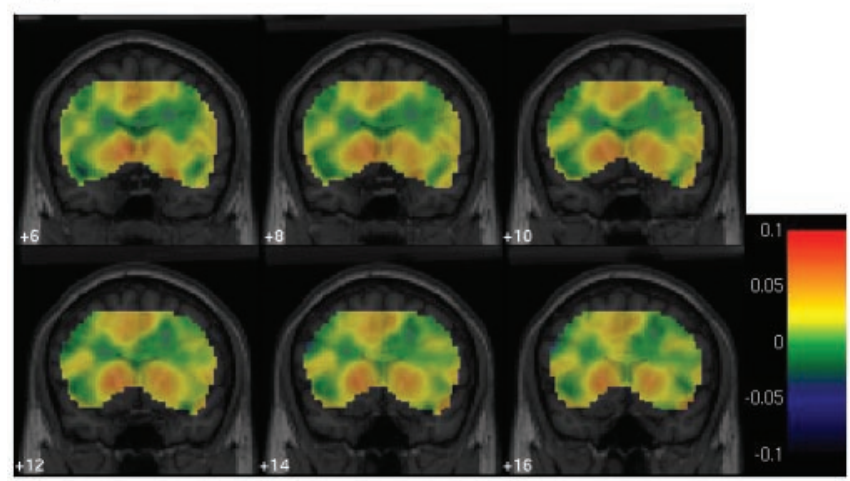

Figure 3. Signal change in the striatum during object switching. $A$, Contrast images (objectswitch trials minus rule-switch trials) with continuous activation values representing percent signal change are shown as a transparent color map superimposed on the MNI template brain (individual brain considered most typical of the 305 brains used to define the MNI standard). $B$, Contrast images (object-rule-switch trials minus rule-switch trials) with continuous activation values (as shown previously).

the mean signal change from each ROI for each switch event relative to the nonswitch baseline events is reported in Table 1. Direct comparison of the critical events of interest revealed a significantly increased signal change in the left VS during objectswitch relative to rule-switch trials (ROI analysis; $t=2.92 ; p=$ $0.005)$. No other effects were observed after either ROI or wholebrain analyses. The reverse contrast, subtracting object-switch events from rule-switch events, did not reveal any significant effects (ROI or whole brain).

Supplementary analyses revealed that the signal change in the left VS was also significantly increased during the combined object-rule-switch events relative to the rule-switch events (ROI analysis; $t=3.31 ; p=0.002$ ), but the increase during the combined object-rule-switch trials relative to the object-switch trials did not reach significance according to our statistical criterion.

To rule out the possibility that we overlooked a signal change that was not present during the combined switch trials but that was nonetheless present during either rule-switch only or objectswitch only trials, we performed a supplementary post hoc analysis. This time, PFC ROIs were selected directly from the contrast comparing all three switch types with the nonswitch trials (at $p<$ $0.05)$. This post hoc ROI analysis also did not reveal any significant differences between rule- and object-switch trials in the PFC (ROIs were at $x, y, z=-3,12,51 ; 33,15,6 ;-30,27,9$; and -42 , $12,9)$.

\section{Behavioral data}

Analysis of RT, error, and omission data revealed significant main effects of trial type for RTs $\left(F_{(3,45)}=70.3 ; p<0.0001\right)$ and 
proportion of errors $\left(F_{(3,45)}=17.8\right.$; $p<$ $0.0001)$ but not for proportion of omissions $\left(F_{(3,45)}=1.2\right)$. Inspection of the data (Fig. 5) revealed that subjects exhibited RT switch costs on all three switch events compared with the baseline nonswitch event, and that error switch costs were particularly pronounced on object-switch and rule-switch events but less so on the object-rule-switch events. Formal planned contrasts revealed that subjects responded significantly more slowly $\left(F_{(1,15)}=391 ; p<0.0001\right)$ and made significantly more errors $\left(F_{(1,15)}=42.4 ; p<\right.$ $0.0001)$ on object-switch trials than on nonswitch trials. Subjects tended to respond faster on rule-switch trials than object-switch trials, but this difference did not reach significance $\left(F_{(1,15)}=4.07 ; p=\right.$ $0.06)$, and there was no difference in terms of errors between these switch trial types.

Subjects made significantly more errors on

rule-switch trials than on object-rule-switch trials $\left(F_{(1,15)}=9.4\right.$; $p=0.008)$, but there was no difference in terms of RTs between these trial types $\left(F_{(1,15)}=1.9 ; p=0.2\right)$.

\section{Discussion}

The present study revealed highly specific BOLD responses in the ventral striatum, which is strongly connected to the OFC (Kemp and Powell, 1970), during object switching. This response was specific to switching between objects and did not extend to switching between abstract task rules when this did not involve the selection of a novel object. Conversely, signal change in the lateral PFC was observed during both abstract rule switching and lower-order switching between specific objects.

These results support the hypothesis that inhibitory mechanisms in frontostriatal systems are organized according to distinct levels of abstraction. Rather than providing support for regional segregation of PFC function, the data suggest separate roles for the VS and lateral PFC. Although a large number of imaging studies have focused on the role of the PFC in inhibitory response control (Konishi et al., 1998; Garavan et al., 1999; Sohn et al., 2000; Sylvester et al., 2003), and a large number of computational models of selection highlight the role of the striatum (Redgrave et al., 1999), only a few studies have compared directly the functions of the PFC and striatum, and these generally have involved clinical populations with nonspecific pathology (Owen et al., 1993; Partiot et al., 1996; Rogers et al., 1998; Dimitrov et al., 1999; Aron et al., 2003; Rieger et al., 2003). The present findings bring together these relatively segregated lines of research and suggest that these structures function in a complementary manner to orchestrate adaptive behavior, with the phylogenetically older striatum operating on concrete, lower-order associations between specific stimuli and responses and the lateral PFC exerting a more general biasing role in the context of changing environments.

The effects are not contaminated by difficulty or reward processing and cannot be accounted for by differential demands for selection, inhibition, working memory, or motor control processes for the following reasons. First, the temporal pattern of the BOLD responses did not match the performance pattern. Thus large RT switch costs were observed for all switch-types, whereas the error switch cost was smallest when subjects switched both
A B
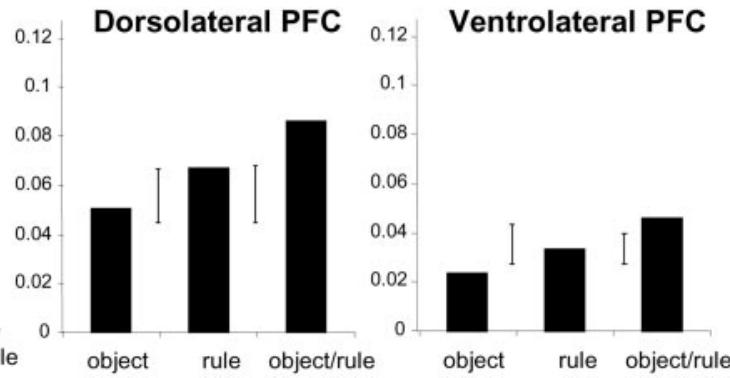

Figure 4. Signal change relative to baseline nonswitch trials. $A$, Percent signal change in the left ventral striatum during 24, 21 ) and nonswitch trials are shown. Error bars represent $S E s$ of the difference.

the object and rule at the same time. The BOLD responses were in fact largest during the combined switch (Fig. 4). Second, feedback was presented depending on accuracy, regardless of trial type, and errors were excluded. Third, although the type of processing was matched across the switch trials, the content of processing was not. During object-switch trials, the previously relevant object had to be inhibited and the newly relevant object had to be selected. During the rule-switch trials, the previously relevant rule had to be inhibited, and the newly relevant rule had to be selected. Fourth, working-memory demands were matched exactly between switch trials, because the need to hold the previously chosen object on-line during the response-stimulus interval was the same on each trial. Fifth, left-right response requirements were matched across all trial types. The behavioral facilitation of switching to a novel object when the rule changed compared with when the rule remained the same is remarkable. This effect is reminiscent of the observation by Rogers and Monsell (1995) that response repetition is facilitatory on nonswitch trials but impairs performance on switch trials. It appears that switching task instruction biases subjects not only to perform a different response but also to shift responding to a different object. Moreover, this bias is accompanied by BOLD increases in both the PFC and VS, leading us to speculate that detection of change at the prefrontal level facilitates change at the striatal level if a lower-order change is required.

The response of the VS during object switching concurs with our previous finding of signal change in the VS during reversal learning (Cools et al., 2002) and also with results from Rogers et al. (2000), who observed activation in the caudate nucleus during reversal learning (relative to intradimensional but not extradimensional shifting). The response of the VS during reversal learning in our previous study (Cools et al., 2002) was accompanied by activation in the VLPFC at anatomical coordinates very similar to those observed here. The present data significantly extend these findings, because they suggest that the role of the striatum is restricted to switching between objects, whereas the role of the lateral PFC generalizes to all forms of switching. Unlike our study, both Nagahama et al. (2001) and Rogers et al. (2000) observed greater signal change in the dorsal PFC during higherorder switching than during lower-order switching. However, higher-order switches in their studies (although not ours) were 
Table 1. Mean signal change relative to baseline nonswitch trials

\begin{tabular}{|c|c|c|c|c|c|}
\hline & Object & Rule & Object-rule & $\mathrm{T}(p)$ & SED (Object-rule) \\
\hline Left VS & 0.022 & 0.002 & 0.043 & $2.92(0.005)$ & 0.007 \\
\hline Right VS & 0.022 & 0.006 & 0.041 & $1.48(0.08)$ & 0.011 \\
\hline Left DS & 0.018 & 0.015 & 0.026 & $0.37(0.84)$ & 0.008 \\
\hline Right DS & 0.018 & 0.015 & 0.025 & $0.41(0.88)$ & 0.011 \\
\hline Left DLPFC & 0.051 & 0.067 & 0.086 & $-0.82(0.79)$ & 0.021 \\
\hline Left VLPFC (BA 11 or 47) & 0.024 & 0.033 & 0.046 & $-0.65(0.74)$ & 0.016 \\
\hline Left VLPFC (BA 45) & 0.043 & 0.049 & 0.056 & $-0.31(0.62)$ & 0.019 \\
\hline $\mathrm{ACC}$ & 0.055 & 0.040 & 0.073 & $1.14(0.14)$ & 0.012 \\
\hline Left PVPFC (BA 44) & 0.055 & 0.048 & 0.068 & $0.38(0.36)$ & 0.018 \\
\hline
\end{tabular}

Percent signal change for each switch trial relative to the baseline nonswitch trials are shown. $t$ and $p$ values represent values for the object-switch minus rule-switch contrast. Note that $p$ values for the reverse contrast (rule-switch minus object-switch contrast) are equal to one minus the values presented here. The SE of the difference (SED) refers to the difference between object- and rule-switch trials. DS, Dorsal striatum; ACC, anterior cingulate cortex; PVPFC, posteroventral prefrontal cortex.

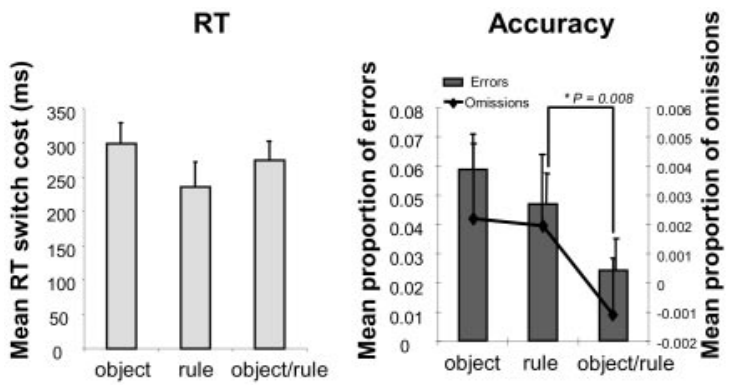

Figure 5. Switch costs. Switch costs were calculated by subtracting performance on baseline nonswitch trials from performance on the three switch trials. RTs in milliseconds and errors and omissions are shown. Error bars represent SEs of the difference.

more difficult than lower-order switches, possibly implying greater demands on working memory, inhibition-selection mechanisms, or greater effort. Alternatively, the different nature of the higher-order shifts (extradimensional shifts in a learning context in previous studies versus conditional task-rule shifts in the present study) may account for the differences in the dorsal PFC.

A role for the striatum in the selection of behaviorally relevant objects is consistent with several computational models of the basal ganglia, many of which emphasize a role in action selection (Graybriel and Kimura, 1995; Jackson and Houghton, 1995; Mink, 1996; Redgrave et al., 1999). The striatum funnels information from the entire cerebral cortex to its output nuclei, which exert a tonic inhibitory influence over thalamocortical and brainstem targets. A focused, context-dependent inhibitory output from the striatum selectively decreases activity in the globus pallidus, which when combined with enhanced tonic inhibition via activity of the subthalamic nucleus in the "indirect" pathway, leads to a finely tuned disinhibition of cortical target areas. This anatomical arrangement is well suited to organize focused disinhibition of desired motor programs and inhibition of competing motor programs (Mink, 1996). A recent study indeed identified "push-pull" pathways that allow the basal ganglia to facilitate visuomotor activity responsible for acquiring a selected target stimulus while suppressing activity associated with potentially competing distractors (Jiang et al., 2003). A role for the striatum in visuomotor selection is supported by fMRI results from Zink et al. (2003) showing striatal activity in response to salient, behaviorally relevant distractors. Several authors (Redgrave et al., 1999) hypothesized that the basal ganglia may be considered to provide a general selection mechanism. However, the present data suggest that the selection computation carried by the VS is restricted to the transformation of specific stimulus-exemplar information into adaptive motor responses, but, unlike the lateral PFC, does not extend to the transformation of abstract representations, such as task rules, into action. This specificity of the actionselection function concurs with findings that patients with Parkinson's disease exhibit switching deficits only when stimuli prime competing responses (Cools et al., 2001; Ravizza and Ciranni, 2002).

Contrary to expectations, no differences were observed between VLPFC and DLPFC responses. Thus, both the VLPFC and DLPFC responded when subjects switched between objects or rules. It should be noted that the unpredictability of the rule switches may have led to substantial rule-switch costs, even on trials when subjects were not switching rules. However, this cannot account for the prefrontal response during object switching relative to nonswitching (Fig. 4), because there is no reason to believe that rule-switch costs would be abolished on nonswitch but not on object-switch trials.

The observation that the lateral PFC is involved whenever behavioral change is needed concurs with the fact that the lateral PFC is robustly activated during Wisconsin Card Sort Test-like shifting, task-switching, go-nogo, and reversal paradigms (Garavan et al., 1999; Konishi et al., 1999; Monchi et al., 2001; Strange et al., 2001; Cools et al., 2002; Swainson et al., 2003). It is also consistent with neurophysiological studies showing that neurons in widespread areas of the PFC encode both simple rules between specific (novel) objects and responses as well as more abstract task rules and stimulus categories (Sakagami and Niki, 1994; Asaad et al., 1998; Freedman et al., 2001; Wallis et al., 2001). Indeed, PFC neurons have been shown to adapt their properties to carry input, action, or reward information relevant to the current task (Rao et al., 1997; Duncan, 2001; Freedman et al., 2001).

The current results do not contradict the proposed ventrodorsal hypothesis, because the response to object switching in the VS [strongly connected to the OFC (Groenewegen et al., 1997)] was not observed in the dorsal striatum, and the signal from the ventromedial PFC was less than robust using current fMRI techniques, as a consequence of field inhomogeneity. Given previously observed associations between the OFC and object alternation (Freedman et al., 1998; Curtis et al., 2000), it remains possible that signal in the medial OFC exhibited a pattern of responding similar to that seen for signal in the VS, but we were unable to detect such changes. Furthermore, the original double dissociation observed by Dias et al. (1996) in marmosets (see Introduction) concerned the OFC versus the lateral PFC, whereas the lateral PFC included both the VLPFC and DLPFC. Nonetheless, the present findings do not provide support for functional segregation within the PFC, consistent with conclusions from 
recent review and meta-analysis (Rushworth and Owen, 1998; Duncan and Owen, 2000). Instead, the data suggest that the lateral PFC responds whenever changes in object relevance or task relevance occur. These findings highlight the different nature of such lateral prefrontal adaptive functioning from the role of the VS (and possibly the OFC) in transforming changes in lowerorder object relevance into adaptive motor responses.

\section{References}

Alexander G, DeLong M, Strick P (1986) Parallel organisation of functionally segregated circuits linking basal ganglia and cortex. Annu Rev Neurosci 9:357-381.

Aron A, Schlaghecken F, Fletcher PC, Bullmore ET, Eimer M, Barker RA, Sahakian BJ, Robbins TW (2003) Inhibition of subliminally primed responses is mediated by the caudate and thalamus: evidence from functional MRI and Huntington's disease. Brain 126:713-723.

Asaad WF, Rainer G, Miller EK (1998) Neural activity in the primate prefrontal cortex during associative learning. Neuron 21:1399-1407.

Brett M, Anton J-L, Valabregue R, Poline J-B (2002) Region of interest analysis using an SPM toolbox. NeuroImage 16:2.

Brown V, Bowman E (2002) Rodent models of prefrontal cortical function. Trends Neurosci 25:340-343.

Chudasama Y, Robbins TW (2003) Dissociable contributions of the orbitofrontal and infralimbic cortex to pavlovian autoshaping and discrimination reversal learning: further evidence for the functional heterogeneity of the rodent prefrontal cortex. J Neurosci 23:8771-8780.

Cools R, Barker RA, Sahakian BJ, Robbins TW (2001) Mechanisms of cognitive set flexibility in Parkinson's disease. Brain 124:2503-2512.

Cools R, Clark L, Owen AM, Robbins TW (2002) Defining the neural mechanisms of probabilistic reversal learning using event-related functional magnetic resonance imaging. J Neurosci 22:4563-4567.

Curtis C, Zald D, Pardo J (2000) Organization of working memory within the human prefrontal cortex: a PET study of self-ordered object working memory. Neuropsychologia 38:1503-1510.

Cusack R, Papadakis N, Martin K, Brett M (2001) A new robust 3D phaseunwrapping algorithm applied to fMRI field maps for the undistortion of EPIs. NeuroImage 13:103.

Dias R, Robbins TW, Roberts AC (1996) Dissociation in prefrontal cortex of affective and attentional shifts. Nature 380:69-72.

Dimitrov M, Grafman J, Soares AHR, Clark K (1999) Concept formation and concept shifting in frontal lesion and Parkinson's disease patients assessed with the California Card Sorting Test. Neuropsychology 13:135-143.

Divac I, Rosvold HE, Szwarcbart MK (1967) Behavioral effects of selective ablation of the caudate nucleus. J Comp Physiol Psychol 63:184-190.

Duncan J (2001) An adaptive coding model of neural function in prefrontal cortex. Nat Rev Neurosci 2:820-829.

Duncan J, Owen AM (2000) Common regions of the human frontal lobe recruited by diverse cognitive demands. Trends Neurosci 23:475-483.

Freedman D, Riesenhuber M, Poggio T, Miller E (2001) Categorical representation of visual stimuli in the primate prefrontal cortex. Science 291:312-316.

Freedman M, Black S, Ebert P, Binns M (1998) Orbitofrontal function, object alternation and perseveration. Cereb Cortex 8:18-27.

Fuster J (1989) The prefrontal cortex: anatomy, physiology, and neuropsychology of the frontal lobe, pp 157-167. New York: Raven.

Garavan H, Ross T, Stein E (1999) Right hemispheric dominance of inhibitory control: an event-related functional MRI study. Proc Natl Acad Sci USA 96:8301-8306.

Graybriel A, Kimura M (1995) Adaptive neural networks in the basal ganglia. In: Models of information processing in the basal ganglia (Houk J, Davis J, Beiser D, eds), pp 103-116. Cambridge, MA: MIT.

Groenewegen H, Wright C, Uylings H (1997) The anatomical relationships of the prefrontal cortex with limbic structures and the basal ganglia. J Psychopharmacol 11:99-106.

Haber SN, Fudge JL, McFarland NR (2000) Striatonigrostriatal pathways in primates form an ascending spiral from the shell to the dorsolateral striatum. J Neurosci 20:2369-2382.

Howell DC (1997) Statistical methods for psychology (Hinrichs C, ed), p 328. Belmont, CA: Wadsworth.

Iversen S, Mishkin M (1970) Perseverative interference in monkeys follow- ing selective lesions of the inferior prefrontal convexity. Exp Brain Res 11:376-386.

Jackson S, Houghton G (1995) Sensorimotor selection and the basal ganglia: a neural network model. In: Information processing in the basal ganglia (Houk J, Davis J, Beiser D, eds), pp 337-367. Cambridge, MA: MIT.

Jiang H, Stein BE, McHaffie JG (2003) Opposing basal ganglia processes shape midbrain visuomotor activity bilaterally. Nature 423:982-986.

Jones B, Mishkin M (1972) Limbic lesions and the problem of stimulusreinforcement associations. Exp Neurol 36:362-377.

Kemp JM, Powell TPS (1970) The cortico-striate projections in the monkey. Brain 93:525-546.

Konishi S, Nakajima K, Uchida I, Kameyama M, Nakahara K, Sekihara K, Miyashita Y (1998) Transient activation of inferior prefrontal cortex during cognitive set shifting. Nat Neurosci 1:80-84.

Konishi S, Nakajima K, Uchida I, Kikyo H, Kameyama M, Miyashita Y (1999) Common inhibitory mechanisms in human inferior prefrontal cortex revealed by event-related functional MRI. Brain 122:981-991.

Milner B (1964) Some effects of frontal lobectomy in man. In: The frontal granular cortex and behaviour (Warren J, Akert K, eds), pp 410-444. New York: McGraw-Hill.

Mink JW (1996) The basal ganglia: focused selection and inhibition of competing motor programs. Prog Neurobiol 50:381-425.

Mishkin M, Vest B, Waxler M, Rosvold HE (1969) A re-examination of the effects of frontal lesions on object alternation. Neuropsychologia 7:357-363.

Monchi O, Petrides M, Petre V, Worsley K, Dagher A (2001) Wisconsin card sorting revisited: distinct neural circuits participating in different stages of the task identified by event-related functional magnetic resonance imaging. J Neurosci 21:7733-7741.

Nagahama Y, Okada T, Katsumi Y, Hayashi T, Yamauchi H, Oyanagi C, Konishi J, Fukuyama H, Shibasaki H (2001) Dissociable mechanisms of attentional control within the human prefrontal cortex. Cereb Cortex 11:85-92.

O’Reilly R, Noelle D, Braver T, Cohen J (2002) Prefrontal cortex and dynamic categorization tasks: representational organization and neuromodulatory control. Cereb Cortex 12:246-257.

Owen AM, Roberts AC, Hodges JR, Summers BA, Polkey CE, Robbins TW (1993) Contrasting mechanisms of impaired attentional set-shifting in patients with frontal lobe damage or Parkinson's disease. Brain 116:1159-1179.

Partiot A, Verin M, Pillon B, Teixeira-Ferreira C, Agid Y, Dubois B (1996) Delayed response tasks in basal ganglia lesions in man: further evidence for a striato-frontal cooperation in behavioural adaptation. Neuropsychologia 34:709-721.

Rao S, Rainer G, Miller E (1997) Integration of what and where in the primate prefrontal cortex. Science 276:821-824.

Ravizza S, Ciranni M (2002) Contributions of the prefrontal cortex and basal ganglia to set shifting. J Cogn Neurosci 14:472-483.

Redgrave P, Prescott TJ, Gurney K (1999) The basal ganglia: a vertebrate solution to the selection problem? Neuroscience 89:1009-1023.

Rieger M, Gauggel S, Burmeister K (2003) Inhibition of ongoing responses following frontal, nonfrontal and basal ganglia lesions. Neuropsychology 17:272-282.

Roberts AC, Wallis JD (2000) Inhibitory control and affective processing in the prefrontal cortex. Cereb Cortex 10:252-262.

Rogers RD, Monsell S (1995) Costs of a predictable switch between simple cognitive tasks. J Exp Psychol 124:207-231.

Rogers RD, Sahakian BJ, Hodges JR, Polkey CE, Kennard C, Robbins TW (1998) Dissociating executive mechanisms of task control following frontal lobe damage and Parkinson's disease. Brain 121:815-842.

Rogers RD, Andrews TC, Grasby PM, Brooks DJ, Robbins TW (2000) Contrasting cortical and subcortical activations produced by attentional-set shifting and reversal learning in humans. J Cogn Neurosci 12:142-162.

Rosvold H (1972) The frontal lobe system: cortical-subcortical interrelationships. Acta Neurobiol Exp (Wars) 32:439-460.

Rushworth MFS, Owen AM (1998) The functional organization of the lateral frontal cortex: conjecture or conjuncture in the electrophysiology literature? Trends Cogn Sci 2:46-53.

Sakagami M, Niki H (1994) Encoding of behavioral significance of visual stimuli by primate prefrontal neurons: relation to relevant task conditions. Exp Brain Res 97:423-436. 
Smith S (2002) Fast robust automated brain extraction. Hum Brain Mapp 17:143-155.

Sohn M, Ursu S, Anderson JR, Stenger VA, Carter CS (2000) The role of prefrontal cortex and posterior parietal cortex in task switching. Proc Natl Acad Sci USA 97:13448-13453.

Stern C, Passingham R (1995) The nucleus accumbens in monkeys (Macaca fascicularis). Exp Brain Res 106:239-247.

Strange BA, Henson RN, Friston KJ, Dolan RJ (2001) Anterior prefrontal cortex mediates rule learning in humans. Cereb Cortex 11:1040-1046.

Swainson R, Cunnington R, Jackson GM, Rorden C, Peters AM, Morris PG, Jackson SR (2003) Cognitive control mechanisms revealed by ERP and fMRI: evidence from repeated task-switching. J Cogn Neurosci 15:785-799.

Sylvester C-YC, Wager TD, Lacey SC, Hernandez L, Nichols TE, Smith EE,
Jonides J (2003) Switching attention and resolving interference: fMRI measures of executive functions. Neuropsychologia 41:357-370.

Talairach J, Tournoux P (1988) Co-planar stereotaxic atlas of the human brain. New York: Thieme.

Tzourio-Mazoyer N, Landeau B, Papthanassiou D, Crivello F, Etard O, Delcroix N, Mazoyer B, Joliot M (2002) Automated anatomical labeling of activations in SPM using a macroscopic anatomical parcellation of the MNI MRI single-subject brain. NeuroImage 15:273-289.

Wallis J, Anderson K, Miller E (2001) Single neurons in prefrontal cortex encode abstract rules. Nature 411:953-956.

Zink CF, Pagnoni G, Martin ME, Dhamala M, Berns GS (2003) Human striatal response to salient nonrewarding stimuli. J Neurosci 23:80928097. 